Review Article

\title{
Hydroxychloroquine Effects on TLR Signalling: Underexposed but Unneglectable in COVID-19
}

\author{
Aliede E. in ' $t$ Veld $\mathbb{D}^{1,2}$ Manon A. A. Jansen ${ }^{10},{ }^{1}$ Luuk C. A. Ciere ${ }^{(D)}$, \\ and Matthijs Moerland $\mathbb{i D}^{1,2}$ \\ ${ }^{1}$ Centre of Human Drug Research, Leiden, Netherlands \\ ${ }^{2}$ Leiden University Medical Center, Leiden, Netherlands \\ Correspondence should be addressed to Matthijs Moerland; mmoerland@chdr.nl
}

Received 12 November 2020; Revised 23 February 2021; Accepted 27 February 2021; Published 11 March 2021

Academic Editor: Herney A. Garcia-Perdomo

Copyright (c) 2021 Aliede E. in 't Veld et al. This is an open access article distributed under the Creative Commons Attribution License, which permits unrestricted use, distribution, and reproduction in any medium, provided the original work is properly cited.

\begin{abstract}
The main basis for hydroxychloroquine (HCQ) treatment in COVID-19 is the compound's ability to inhibit viral replication in vitro. HCQ also suppresses immunity, mainly by interference in TLR signalling, but reliable clinical data on the extent and nature of HCQ-induced immunosuppression are lacking. Here, we discuss the mechanistic basis for the use of HCQ against SARS-CoV-2 in a prophylactic setting and in a therapeutic setting, at different stages of the disease. We argue that the clinical effect of prophylactic or therapeutic HCQ treatment in COVID-19 depends on the balance between inhibition of viral replication, immunosuppression, and off-target side effects, and that the outcome is probably dependent on disease stage and disease severity. This is supported by the initial outcomes of the well-designed randomized controlled trials: so far, evidence for a beneficial effect of HCQ treatment for COVID-19 is weak and conflicting.
\end{abstract}

\section{Introduction}

Hydroxychloroquine sulfate (HCQ, Figure 1) is a less toxic derivative of the antimalarial drug chloroquine (CQ). Besides the use as antimalarial drug, HCQ is also prescribed for the treatment of several different autoimmune diseases such as rheumatoid arthritis, juvenile idiopathic arthritis, and systemic lupus erythematosus. The compound has been evaluated extensively in an ever-increasing number of clinical trials as treatment modality to fight SARS-CoV-2 infection and also in a prophylactic setting. The insight we are aiming to provide in this paper is whether the effects of HCQ use on SARS-CoV-2 infection align with the predicted effects of HCQ. Can the molecular activities of the drug, in particular its direct immunosuppressive activities, predict the effect on SARS-CoV-2 infection? We advocate that especially these immunosuppressive effects ultimately determine the clinical outcome, while so far they have remained largely underexposed in clinical trials evaluating HCQ effects on COVID-19.
The main reason why HCQ initially emerged as potential treatment in COVID-19 was because of its in vitro antiviral properties against several RNA viruses, including SARSCoV-1 and -2 [1-4]. In addition, HCQ possesses immunosuppressive properties that may be beneficial in dampening the derailed immune response in later stages of SARS-CoV2 infection [5]. Based on these pharmacological activities, HCQ was considered to be a promising drug to combat COVID-19, at least until the moment an effective vaccine would become available. In spring 2020, this even urged some governments to recommend prophylactic HCQ use, for example, by the Indian Council of Medicinal Research [6] and repeatedly by President Trump in White House briefings. This was remarkable, because at that moment in time, conclusive data from large, randomized, and wellmonitored clinical trials on the preventive or therapeutic efficacy of HCQ in COVID-19 were pending. The outcomes of initial clinical studies evaluating HCQ effects in COVID-19 were not convincing, also because many studies suffered 
<smiles>CCN(CCO)CCCC(C)Nc1ccnc2cc(Cl)ccc12</smiles>

FIGURE 1: Chemical structure of hydroxychloroquine sulfate.

from major methodological limitations and decent peer review of study reports was complicated by time constraints. This has been extensively outlined in earlier reviews [7] and was breaking news when two papers in The Lancet and New England Journal of Medicine were retracted [8, 9]. Six months later, the results of the first randomized controlled trials have been published, and overall, they are disappointing. So far, there is no convincing proof for beneficial effects of HCQ, neither in a therapeutic setting nor in a postexposure prophylactic setting [10].

A complicating factor for the evaluation of HCQ's effects on COVID-19 has been the highly variable pathophysiology, within an individual patient over time, but also between patients [11]. HCQ's inhibiting effect on SARS-CoV-2 replication, based on in vitro evidence, would be beneficial at any stage of the disease, in any population (being it noninfected subjects, asymptomatic patients, or severe patients). However, this is not equally self-evident for the compound's immunosuppressive effects, as we will outline later in this manuscript. Importantly, despite extensive mechanistic evidence based on in vitro experiments, reliable clinical data on the extent and nature of HCQ-induced immunosuppression are lacking.

This article discusses the mechanistic basis for the use of HCQ against SARS-CoV-2 in a prophylactic setting and in a therapeutic setting, at different stages of the disease. The focus lies on HCQ's immunosuppressive effects, since we advocate that especially this aspect is largely underexposed in recent clinical trials evaluating HCQ effects on COVID19. A nonsystematic review of published literature was performed, mainly PubMed-based, to build this mechanistic basis. This article only discusses HCQ, since this compound suffers less from side effects, drug-drug interactions, and toxicity than its parent compound chloroquine, while their pharmacological activities are well comparable [12].

\section{Immunosuppressive Effects of HCQ}

The basis for HCQ's use in autoimmune diseases is its wide range of immunosuppressive properties (Figure 2). HCQ accumulates in the lysosomes where it increases the $\mathrm{pH}$ and inhibits the enzymatic activity in both lysosomes and autophagosomes. Since these organelles play an important role in antigen processing and MHC class II presentation, a rise in lysosomal $\mathrm{pH}$ indirectly inhibits the immune response to both intracellular and extracellular antigens [13].

Lysosomal accumulation of HCQ does not only result in a $\mathrm{pH}$ increase, but also directly affect endosomal TLR signalling triggered by nucleic acids. The endosomal TLRs (i.e.,
TLR3, TLR7, TLR8, and TLR9) play an important role in the innate immune response by recognizing doublestranded RNA, single-stranded RNA, and CpG motifs in viral DNA [14]. HCQ can bind nucleic acids within the endosome, thereby preventing interaction of the endosomal TLRs with their ligands, inhibiting subsequent TLR activation. Downstream innate immune responses are dampened, such as IFN- $\alpha$ and TNF production by plasmacytoid dendritic cells $[15,16]$. In addition, the adaptive immune response is impaired by HCQ effects on B cell differentiation and cytokine production $[17,18]$. Moreover, HCQ inhibits T cell activation, proliferation, and cytokine production by inhibiting intracellular calcium and mobilization and subsequent NFAT signalling $[19,20]$ and apoptosis in CD45RO+ memory and effector $\mathrm{T}$ cells by inhibiting autophagy [21].

The majority of the mechanistic work on HCQ's immunosuppressive activity has been performed in cell lines. Experimental evidence for immune suppression by HCQ in primary human cells is scarce. Some publications are available describing HCQ effects on innate immune responses in human whole blood, peripheral blood mononuclear cells, or $\mathrm{T}$ cells, with TLR-mediated cytokine production, or $\mathrm{T}$ cell activation and proliferation as endpoint [22-27]. Most experiments used HCQ concentrations largely exceeding expected circulating concentrations in vivo after prophylactic or therapeutic dosing. Moreover, with one exception, none of the papers provides a decent HCQ concentration-effect relationship, so an IC50 for HCQ's immunosuppressive activities cannot be estimated. Interestingly, HCQ's IC50 for inhibition of SARS-CoV-2 replication (4-17 $\mu \mathrm{M})$ [28] appears to exceed HCQ concentrations effectively inhibiting TLR responses in vitro $(3 \mu \mathrm{M})[24,27]$, which means that it will be difficult to inhibit viral replication without impairing the immune system.

\section{Mechanistic Support for HCQ Use in COVID-} 19

3.1. Prophylactic Setting. Cell entry by SARS-CoV-2 is thought to be similar to SARS-CoV entry, being mediated by spike (S) protein binding to angiotensin-converting enzyme 2 (ACE2) $[29,30]$. In silico predictions showed that HCQ prevents the cellular binding and entering of SARS$\mathrm{CoV}-2$ virus particles, by interfering with sialic acids and surface gangliosides [31]. Based on this pharmacological activity, prophylactic HCQ treatment could theoretically be beneficial and prevent SARS-CoV-2 infection in vulnerable populations or populations professionally exposed to COVID-19 patients.

Upon cell entry, SARS-CoV-2 is likely recognized by TLR3, TLR4, TLR7, TLR8, and RIG-1 [32], resulting in a type I IFN response which is crucial for an efficient adaptive antiviral response [33]. HCQ suppresses parts of the immune system that are essential in fighting infections, including TLR signalling and type I IFN production. In previous SARS-CoV and MERS-CoV outbreaks, downregulation of IFNs by coronavirus proteins strongly correlated with worse disease progression and increased lethality [34]. Cell and animal models of SARS-CoV-2 infection and transcriptional 


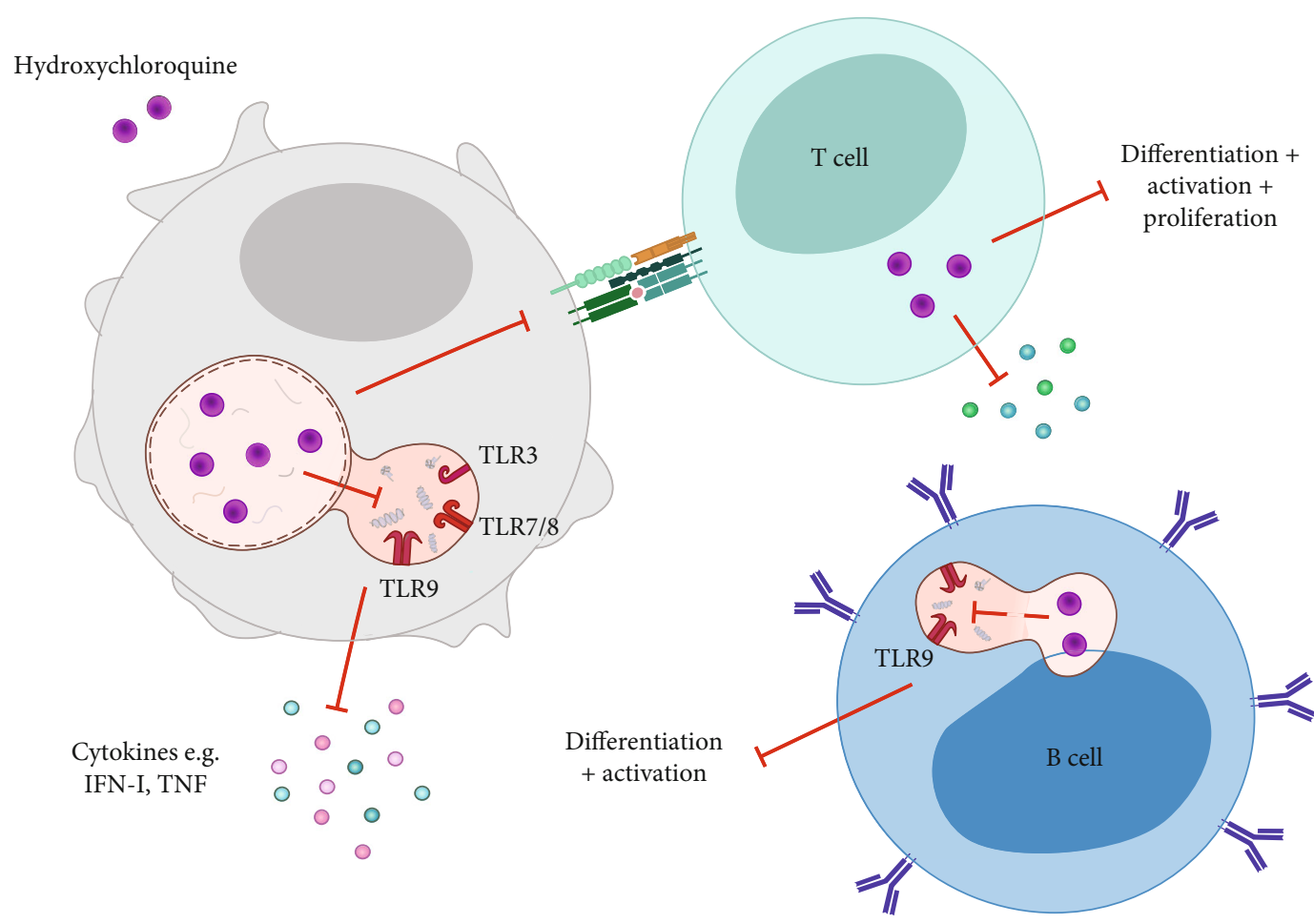

FIGURE 2: Immunosuppressive effects of HCQ. Hydroxychloroquine affects both the innate and adaptive immune system. By accumulating in the lysosome and autophagosome, the $\mathrm{pH}$ is increased causing an inhibition of MHC-II antigen presentation and subsequent $\mathrm{T}$ cell activation. In addition, HCQ accumulation abrogates viral recognition by endosomal TLRs, resulting in a decrease of the antiviral innate immune response (i.e., IFN-I production). Moreover, HCQ can also directly affect the adaptive immune system through inhibition of T and B cell differentiation and activation.

and serum profiling of COVID-19 patients revealed an imbalanced host response with low levels of types I and III IFNs [35]. Early IFN signalling was protective in SARS$\mathrm{CoV}$-1-infected mice, whereas delayed IFN signalling was detrimental leading to severe disease progression and related lethal pneumonia [36].

The importance of TLR signalling in viral defence has been well established in SARS-CoV-1 mouse models. Both TLR3 and TLR4 deficient mice are more susceptible to SARS-CoV-1 infections [37]. Murine MyD88 or TRIF deficiency, which are downstream signalling molecules shared by multiple TLRs, resulted in a mortality rate of over $90 \%$ upon experimental infection with SARS-CoV-1, which is usually nonlethal in immunocompetent mice [37, 38]. HCQ abrogates endosomal acidification thereby reducing endosomal TLR activation [22], but interestingly, enough data confirming this HCQ effect on endosomal TLRs in primary human cells are scarce. Since the relationship between HCQ dose/concentration and level of immunosuppression remains largely unexplored in primary human immune cells, it is difficult to estimate the effect of prophylactic HCQ treatment regimens on the innate immune response. If HCQ's immunosuppressive IC50s would fall in the concentration range reached after prophylactic HCQ treatment, endosomal TLR responses, type I IFN production, and T and B cell activation and proliferation could be impaired in vivo. Theoretically, this could result in an increased viral infection risk, including SARS-CoV-2 infection. On the other hand, HCQ use in rheumatoid arthritis patients is not associated with an increased infection risk $[39,40]$. So far, prophylactic HCQ studies did not show clinical benefit of HCQ administration $[41,42]$.

Next to mechanistic arguments, the fact that long-term HCQ use comes with side effects further fuels doubts about prophylactic use of HCQ. Retinal toxicity, cardiac disease, neuromyopathy (reversible), dermatological manifestations, gastrointestinal and hematological changes, and hearing abnormalities have been reported upon long-term HCQ treatment, amongst others [43-45]. Such side effects could be avoided by local HCQ administration, for example, by inhalation.

3.2. Therapeutic Setting. Although our understanding of the pathophysiology continues to increase on a daily basis, it is clear that COVID-19 is a highly heterogenous disease. With increased disease severity, the complexity of the pathophysiology grows $[32,46]$. Since many excellent reviews are available in the public domain, this manuscript does not revisit COVID-19 pathophysiology and disease progression. Instead, it discusses the alignment between HCQ's mechanism of action and disease stage: how could specific pharmacological activities of HCQ theoretically affect COVID-19's pathophysiology at a particular disease stage? As guidance, the disease progression has been separated into three stages: stage 1-virus entry and replication in the airway cells (days $0-2)$, stage 2-activation of innate immunity in the lung 


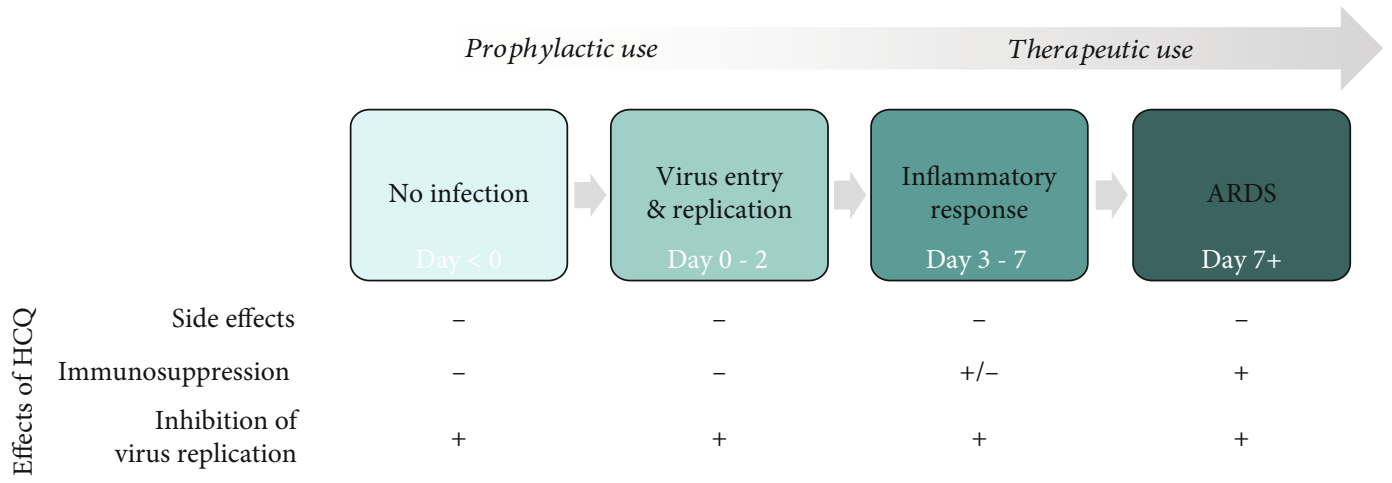

Figure 3: Theoretical effects of HCQ at different stages of SARS-CoV-2 infection. Potential HCQ effects on COVID-19 are schematically presented over the course of the disease, ranging from prophylactic use in uninfected subjects to therapeutic use in acute respiratory distress syndrome (ARDS) in severe patients. A beneficial HCQ effect is indicated with "+" and a detrimental HCQ effect with "-." The stages of SARS-CoV-2 infection are indicated in green. Stage 0 - no infection, stage 1 -virus entry and replication in the airway cells, stage 2 - activation of innate and adaptive immune system, and stage 3-ARDS.

(maladaptive inflammatory response, days 3-7), and stage 3-acute respiratory distress syndrome (ARDS, > day 7 [47]). Obviously, the clinical presentation of COVID-19 varies between patients from asymptomatic to mild, moderate, and severe, and not all patients develop advanced disease stages.

When discussing potential effects of HCQ treatment in a therapeutic setting, most papers focus on the off-target side effects of HCQ, specifically potentially severe cardiac disorders such as QT segment prolongation. However, safety concerns related to the short-term use of HCQ (i.e., regimens of 1 month) are probably limited, as demonstrated by a recently published (nonpeer reviewed) international study in more than 900,000 HCQ-treated patients [48]. We advocate that one of HCQ's pharmacological activities, namely, its immunosuppressive effect, is critical when considering HCQ as potential treatment modality for COVID-19. Surprisingly, HCQ's exact molecular mechanism of action has remained largely neglected in considerations on therapeutic HCQ use for COVID19. Therefore, we discuss in the next sections how HCQ's pharmacological activities could be beneficial or detrimental, at different disease stages (stages 1-3, see above) and in different disease severities (asymptomatic, mild, moderate, and severe) (Figure 3).

For therapeutic treatment, the first stage (days 0-2 of infection) is irrelevant, since patients are asymptomatic and viral titers may be low [49], so patients in this stage of the disease are untreated or fall in the prophylactic treatment category (see previous section). HCQ treatment theoretically could be beneficial in the next stages of the disease (stage 2: days 3-7 and stage 3: >day 7), when the innate immune response in the lungs starts to evolve and ultimately culminates in respiratory impairment and multiorgan failure. The drug may not only inhibit virus replication, but also suppress TLR-mediated cytokine responses and overactivation and apoptosis of lymphocytes, processes that are observed in severe COVID-19 [50, 51]. Especially prevention of a cytokine storm is critical since this is a major factor driving multiorgan failure, ARDS, disseminated intravascular coagulation, and the resulting high mortality. Taken together, HCQ treatment in progressed COVID-19 is mechanistically supported by HCQ's pharmacological activities.

Obviously, progressed disease as outlined above (stages 2 and 3) only applies to moderate to severe COVID-19 patients. The large majority of COVID-19 patients only suffers from mild disease or even remains asymptomatic [52]. These patients have a low viral load, develop an efficient type I IFN response, produce virus-neutralizing antibodies, and do not develop a maladaptive inflammatory response [53]. Since it is especially the latter response that could be targeted by HCQ's immunosuppressive activity, the question arises whether HCQ treatment is rational in asymptomatic or mild patients. On one hand, one could argue that HCQ-dependent inhibition of viral replication (though not clinically proven) is important, independent of disease stage. Moreover, HCQ-dependent immunosuppression may prevent mild disease turning into inflammation-driven moderate/severe disease. On the other hand, in the early disease stage, it is important that the virus-specific anti-SARS-CoV-2 response is driven by an efficient antiviral innate immune response, and especially this response may be significantly impaired upon HCQ treatment. The net result of HCQ treatment will depend on the balance between these two pharmacological activities. The outcome of therapeutic studies has shown that HCQ treatment overall does not seem to reduce mortality, improve clinical scores, or suppress viral load in moderate to severe COVID-19 patients [54-56]. However, low-dose HCQ treatment $(<2.5 \mathrm{~g}$ in total) was associated with a reduced risk of intensive care unit admission and lower mortality rates $[57,58]$. HCQ's clinical beneficial effects may depend on the inflammatory status of the patient: chronic low-dose HCQ treatment of a large cohort of rheumatic patients coincided with reduced mortality following SARSCoV-2 infection [59], and another study reported a therapeutic benefit of HCQ treatment in patients with elevated Creactive protein levels [60]. These reports are mechanistically in line with the immunosuppressive activities of HCQ, as outlined above. 


\section{Conclusion}

Immunosuppression by HCQ, via interference in endosomal TLR signalling, has remained largely underexposed in the public debate, while it may be a critical factor for the (lack of?) clinical efficacy of HCQ in COVID-19. Experimental evidence for immune suppression by HCQ in primary human cells is scarce, which is surprising for such an old drug. Clinical trials evaluating HCQ as COVID-19 treatment did not include readout measures to study this immunosuppressive effect of HCQ. As a result, the extent of immunosuppression by HCQ cannot be reliably estimated in vivo. If systemic or local HCQ concentrations would be sufficiently high to suppress key components of the innate immune response, this could translate into a clinical benefit. The other side of the coin is that in mild COVID-19 patients or in a prophylactic setting, immunosuppression by HCQ could have a detrimental effect, since an efficient virus-specific anti-SARS-CoV-2 response depends on a robust antiviral innate immune response. We argue that ultimately the clinical effect of HCQ treatment in COVID-19 depends on the balance between inhibition of viral replication, immunosuppression, and off-target side effects (which have been extensively evaluated recently, within and outside the setting of COVID-19 treatment or prevention, in [61, 62], and are as such not the topic of this article). The outcome of this balance is probably dependent on disease stage and disease severity (Figure 3). This is supported by the initial outcomes of the well-designed randomized controlled trials: so far, evidence for a beneficial effect of HCQ treatment for COVID-19 is weak and conflicting.

\section{Conflicts of Interest}

The authors declare that there is no conflict of interest regarding the publication of this paper.

\section{References}

[1] A. Savarino, J. R. Boelaert, A. Cassone, G. Majori, and R. Cauda, "Effects of chloroquine on viral infections: an old drug against today's diseases?," The Lancet Infectious Diseases, vol. 3, no. 11, pp. 722-727, 2003.

[2] T. S. Mele and P. F. Halloran, "The use of mycophenolate mofetil in transplant recipients," Immunopharmacology, vol. 47, no. 2-3, pp. 215-245, 2000.

[3] E. Keyaerts, L. Vijgen, P. Maes, J. Neyts, and M. V. Ranst, "In vitro inhibition of severe acute respiratory syndrome coronavirus by chloroquine," Biochemical and Biophysical Research Communications, vol. 323, no. 1, pp. 264-268, 2004.

[4] M. J. Vincent, E. Bergeron, S. Benjannet et al., "Chloroquine is a potent inhibitor of SARS coronavirus infection and spread," Virology Journal, vol. 2, no. 1, p. 69, 2005.

[5] X. Yao, F. Ye, M. Zhang et al., "In vitro antiviral activity and projection of optimized dosing design of hydroxychloroquine for the treatment of severe acute respiratory syndrome coronavirus 2 (SARS-CoV-2)," Clinical Infectious Diseases, vol. 71, no. 15 , pp. $732-739,2020$.
[6] Indian National Taskforce for COVID19, "Advisory on the use of hydroxy-chloroquine as prophylaxis for SARS-CoV-2 infection," 2020.

[7] S. Das, S. Bhowmick, S. Tiwari, and S. Sen, "An updated systematic review of the therapeutic role of hydroxychloroquine in coronavirus disease-19 (COVID-19)," Clinical Drug Investigation, vol. 40, pp. 1-11, 2020.

[8] M. R. Mehra, F. Ruschitzka, and A. N. Patel, "Retraction-hydroxychloroquine or chloroquine with or without a macrolide for treatment of COVID-19: a multinational registry analysis," The Lancet, vol. 395, 2020.

[9] M. R. Mehra, S. S. Desai, S. Kuy, T. D. Henry, and A. N. Patel, "Retraction: cardiovascular disease, drug therapy, and mortality in Covid-19," New England journal of medicine, vol. 382, no. 26, p. 2582, 2020.

[10] C. Rughiniş, L. Dima, and S. Vasile, "Hydroxychloroquine and COVID-19: lack of efficacy and the social construction of plausibility," American Journal of Therapeutics, vol. 27, no. 6, pp. e573-e583, 2020.

[11] X. Zhang, Y. Tan, Y. Ling et al., "Viral and host factors related to the clinical outcome of COVID-19," Nature, vol. 583, no. 7816, pp. 437-440, 2020.

[12] A. Gasmi, M. Peana, S. Noor et al., "Chloroquine and hydroxychloroquine in the treatment of COVID-19: the never-ending story," Applied Microbiology and Biotechnology, vol. 105, no. 4, pp. 1333-1343, 2021.

[13] E. Schrezenmeier and T. Dörner, "Mechanisms of action of hydroxychloroquine and chloroquine: implications for rheumatology," Nature Reviews Rheumatology, vol. 16, no. 3, pp. 155-166, 2020.

[14] T. Kawasaki and T. Kawai, "Toll-like receptor signaling pathways," Frontiers in Immunology, vol. 5, p. 461, 2014.

[15] K. Sacre, L. A. Criswell, and J. M. McCune, "Hydroxychloroquine is associated with impaired interferon-alpha and tumor necrosis factor-alpha production by plasmacytoid dendritic cells in systemic lupus erythematosus," Arthritis Research \& Therapy, vol. 14, no. 3, p. R155, 2012.

[16] I. L. A. Bodewes, J.-E. Gottenberg, C. G. van Helden-Meeuwsen, X. Mariette, and M. A. Versnel, "Hydroxychloroquine treatment downregulates systemic interferon activation in primary Sjögren's syndrome in the JOQUER randomized trial," Rheumatology, vol. 59, pp. 107-111, 2019.

[17] M. Torigoe, K. Sakata, A. Ishii, S. Iwata, S. Nakayamada, and Y. Tanaka, "Hydroxychloroquine efficiently suppresses inflammatory responses of human class-switched memory B cells via Toll-like receptor 9 inhibition," Clinical immunology (Orlando, Fla), vol. 195, pp. 1-7, 2018.

[18] S. Brauner, L. Folkersen, M. Kvarnström et al., "H1N1 vaccination in Sjögren's syndrome triggers polyclonal B cell activation and promotes autoantibody production," Annals of the Rheumatic Diseases, vol. 76, no. 10, pp. 1755-1763, 2017.

[19] F. D. Goldman, A. L. Gilman, C. Hollenback, R. M. Kato, B. A. Premack, and D. J. Rawlings, "Hydroxychloroquine inhibits calcium signals in $\mathrm{T}$ cells: a new mechanism to explain its immunomodulatory properties," Blood, vol. 95, no. 11, pp. 3460-3466, 2000.

[20] S. F. Wu, C. B. Chang, J. M. Hsu et al., "Hydroxychloroquine inhibits CD154 expression in CD4(+) T lymphocytes of systemic lupus erythematosus through NFAT, but not STAT5, signaling," Arthritis Research \& Therapy, vol. 19, no. 1, p. $183,2017$. 
[21] J. van Loosdregt, R. Spreafico, M. Rossetti, B. J. Prakken, M. Lotz, and S. Albani, "Hydroxychloroquine preferentially induces apoptosis of CD45RO+ effector T cells by inhibiting autophagy: a possible mechanism for therapeutic modulation of T cells," The Journal of Allergy and Clinical Immunology, vol. 131, pp. 1443-1446.e1, 2013.

[22] A. Kuznik, M. Bencina, U. Svajger, M. Jeras, B. Rozman, and R. Jerala, "Mechanism of endosomal TLR inhibition by antimalarial drugs and imidazoquinolines," Journal of Immunology (Baltimore, Md: 1950), vol. 186, pp. 4794-4804, 2011.

[23] A. Gardet, A. Pellerin, C. A. McCarl et al., "Effect of in vivo hydroxychloroquine and ex vivo anti-BDCA2 mAb treatment on $\mathrm{pDC}$ IFN $\alpha$ production from patients affected with cutaneous lupus erythematosus," Frontiers in Immunology, vol. 10, p. 275, 2019.

[24] M. Zeidi, H. J. Kim, and V. P. Werth, "Increased myeloid dendritic cells and TNF- $\alpha$ expression predicts poor response to hydroxychloroquine in cutaneous lupus erythematosus," The Journal of Investigative Dermatology, vol. 139, no. 2, pp. 324$332,2019$.

[25] J. C. Silva, H. A. Mariz, L. F. Rocha Jr. et al., "Hydroxychloroquine decreases Th17-related cytokines in systemic lupus erythematosus and rheumatoid arthritis patients," Clinics (Sao Paulo, Brazil), vol. 68, no. 6, pp. 766-771, 2013.

[26] R. L. Schmidt, S. Jutz, K. Goldhahn et al., "Chloroquine inhibits human $\mathrm{CD}^{+}$T-cell activation by AP-1 signaling modulation," Scientific Reports, vol. 7, no. 1, article 42191, 2017.

[27] P. Alves, M. M. Bashir, M. Wysocka, M. Zeidi, R. Feng, and V. P. Werth, "Quinacrine suppresses tumor necrosis factor- $\alpha$ and IFN- $\alpha$ in dermatomyositis and cutaneous lupus erythematosus," The Journal of Investigative Dermatology Symposium Proceedings, vol. 18, no. 2, pp. S57-s63, 2017.

[28] J. Liu, R. Cao, M. Xu et al., "Hydroxychloroquine, a less toxic derivative of chloroquine, is effective in inhibiting SARSCoV-2 infection in vitro," Cell Discovery, vol. 6, no. 1, p. 16, 2020.

[29] M. Hoffmann, H. Kleine-Weber, S. Schroeder et al., "SARSCoV-2 cell entry depends on ACE2 and TMPRSS2 and is blocked by a clinically proven protease inhibitor," Cell, vol. 181, no. 2, pp. 271-80.e8, 2020.

[30] W. Li, M. J. Moore, N. Vasilieva et al., "Angiotensin-converting enzyme 2 is a functional receptor for the SARS coronavirus," Nature, vol. 426, no. 6965, pp. 450-454, 2003.

[31] J. Fantini, C. Di Scala, H. Chahinian, and N. Yahi, "Structural and molecular modelling studies reveal a new mechanism of action of chloroquine and hydroxychloroquine against SARS-CoV-2 infection," International Journal of Antimicrobial Agents, vol. 55, no. 5, article 105960, 2020.

[32] N. Vabret, G. J. Britton, C. Gruber et al., "Immunology of COVID-19: current state of the science," Immunity, vol. 52, no. 6, pp. 910-941, 2020.

[33] C. E. Samuel, “Antiviral actions of interferons," Clinical Microbiology Reviews, vol. 14, pp. 778-809, 2001.

[34] E. de Wit, N. van Doremalen, D. Falzarano, and V. J. Munster, "SARS and MERS: recent insights into emerging coronaviruses," Nature Reviews Microbiology, vol. 14, no. 8, pp. 523-534, 2016.

[35] D. Blanco-Melo, B. E. Nilsson-Payant, W. C. Liu et al., "Imbalanced host response to SARS-CoV-2 drives development of COVID-19," Cell, vol. 181, no. 5, pp. 1036-1045.e9, 2020.
[36] R. Channappanavar, A. R. Fehr, R. Vijay et al., "Dysregulated type I interferon and inflammatory monocyte-macrophage responses cause lethal pneumonia in SARS-CoV-infected mice," Cell Host \& Microbe, vol. 19, no. 2, pp. 181-193, 2016.

[37] A. L. Totura, A. Whitmore, S. Agnihothram et al., "Toll-like receptor 3 signaling via TRIF contributes to a protective innate immune response to severe acute respiratory syndrome coronavirus infection," mBio, vol. 6, no. 3, p. e00638, 2015.

[38] T. Sheahan, T. E. Morrison, W. Funkhouser et al., "MyD88 is required for protection from lethal infection with a mouseadapted SARS-CoV," PLoS Pathogens, vol. 4, no. 12, article e1000240, 2008.

[39] F. Wolfe, L. Caplan, and K. Michaud, “Treatment for rheumatoid arthritis and the risk of hospitalization for pneumonia: associations with prednisone, disease-modifying antirheumatic drugs, and anti-tumor necrosis factor therapy," Arthritis and Rheumatism, vol. 54, no. 2, pp. 628-634, 2006.

[40] S. Bernatsky, M. Hudson, and S. Suissa, "Anti-rheumatic drug use and risk of serious infections in rheumatoid arthritis," Rheumatology (Oxford, England), vol. 46, pp. 1157-1160, 2007.

[41] B. S. Abella, E. L. Jolkovsky, B. T. Biney et al., "Efficacy and safety of hydroxychloroquine vs placebo for pre-exposure SARS-CoV-2 prophylaxis among health care workers: a randomized clinical trial," JAMA Internal Medicine, vol. 181, no. 2, pp. 195-202, 2021.

[42] D. R. Boulware, M. F. Pullen, A. S. Bangdiwala et al., "A randomized trial of hydroxychloroquine as postexposure prophylaxis for Covid-19," The New England Journal of Medicine, vol. 383, no. 6, pp. 517-525, 2020.

[43] H. Yogasundaram, B. N. Putko, J. Tien et al., "Hydroxychloroquine-induced cardiomyopathy: case report, pathophysiology, diagnosis, and treatment," Canadian Journal of Cardiology, vol. 30, no. 12, pp. 1706-1715, 2014.

[44] J. B. Kwon, A. Kleiner, K. Ishida, J. Godown, E. Ciafaloni, and R. J. Looney Jr., "Hydroxychloroquine-induced myopathy," Journal of Clinical Rheumatology, vol. 16, no. 1, pp. 28-31, 2010.

[45] I. Ben-Zvi, S. Kivity, P. Langevitz, and Y. Shoenfeld, "Hydroxychloroquine: from malaria to autoimmunity," Clinical Reviews in Allergy \& Immunology, vol. 42, no. 2, pp. 145-153, 2012.

[46] A. K. Azkur, M. Akdis, D. Azkur et al., "Immune response to SARS-CoV-2 and mechanisms of immunopathological changes in COVID-19," Allergy, vol. 75, no. 7, pp. 15641581, 2020.

[47] A. M. Risitano, D. C. Mastellos, M. Huber-Lang et al., "Complement as a target in COVID-19?," Nature Reviews Immunology, vol. 20, no. 6, pp. 343-344, 2020.

[48] J. C. E. Lane, J. Weaver, K. Kostka et al., "Safety of hydroxychloroquine, alone and in combination with azithromycin, in light of rapid wide-spread use for COVID-19: a multinational, network cohort and self-controlled case series study," med Rxiv, vol. 2, 20202020.04.08.20054551.

[49] N. Sethuraman, S. S. Jeremiah, and A. Ryo, "Interpreting diagnostic tests for SARS-CoV-2," Journal of the American Medical Association, vol. 323, no. 22, pp. 2249-2251, 2020.

[50] F. Wang, J. Nie, H. Wang et al., "Characteristics of peripheral lymphocyte subset alteration in COVID-19 pneumonia," The Journal of Infectious Diseases, vol. 221, no. 11, pp. 17621769, 2020. 
[51] E. Prompetchara, C. Ketloy, and T. Palaga, "Immune responses in COVID-19 and potential vaccines: lessons learned from SARS and MERS epidemic," Asian Pacific Journal of Allergy and Immunology, vol. 38, no. 1, pp. 1-9, 2020.

[52] World Health Organization, "Report of the WHO-China joint mission on coronavirus disease 2019 (COVID-19)," 2020.

[53] F. Wang, H. Hou, Y. Luo et al., "The laboratory tests and host immunity of COVID-19 patients with different severity of illness," JCI Insight, vol. 5, no. 10, 2020.

[54] R. J. Ulrich, A. B. Troxel, E. Carmody et al., "Treating COVID19 with hydroxychloroquine (TEACH): a multicenter, doubleblind randomized controlled trial in hospitalized patients," Open Forum Infectious Diseases, vol. 7, no. 10, article ofaa 446, 2020.

[55] T. Fiolet, A. Guihur, M. E. Rebeaud, M. Mulot, N. PeifferSmadja, and Y. Mahamat-Saleh, "Effect of hydroxychloroquine with or without azithromycin on the mortality of coronavirus disease 2019 (COVID-19) patients: a systematic review and meta-analysis," Clinical Microbiology and Infection, vol. 27, 2020.

[56] K. S. Faíco-Filho, D. D. Conte, L. K. de Souza Luna, J. M. A. Carvalho, A. H. S. Perosa, and N. Bellei, "No benefit of hydroxychloroquine on SARS-CoV-2 viral load reduction in noncritical hospitalized patients with COVID-19," Brazilian journal of microbiology: [publication of the Brazilian Society for Microbiology], vol. 51, pp. 1-5, 2020.

[57] N. Dauby and E. Bottieau, "The unfinished story of hydroxychloroquine in COVID-19: the right anti-inflammatory dose at the right moment?," International Journal of Infectious Diseases, vol. 103, 2020.

[58] A. J. J. Lammers, R. M. Brohet, R. E. P. Theunissen et al., "Early hydroxychloroquine but not chloroquine use reduces ICU admission in COVID-19 patients," International Journal of Infectious Diseases, vol. 101, pp. 283-289, 2020.

[59] C. A. Gentry, M. B. Humphrey, S. K. Thind, S. C. Hendrickson, G. Kurdgelashvili, and R. J. Williams 2nd., "Long-term hydroxychloroquine use in patients with rheumatic conditions and development of SARS-CoV-2 infection: a retrospective cohort study," The Lancet Rheumatology, vol. 2, no. 11, pp. e689e697, 2020.

[60] A. D. Castelnuovo, S. Costanzo, A. Antinori et al., "Use of hydroxychloroquine in hospitalised COVID-19 patients is associated with reduced mortality: findings from the observational multicentre Italian CORIST study," European Journal of Internal Medicine, vol. 82, pp. 38-47, 2020.

[61] A. E. Maraolo and A. Grossi, "Safety of hydroxychloroquine for treatment or prevention of SARS-CoV-2 infection: a rapid systematic review and meta-analysis of randomized clinical trials," Immunity, Inflammation and Disease, vol. 9, no. 1, pp. 31-36, 2021.

[62] S. Celotto, N. Veronese, M. Barbagallo et al., “An umbrella review of systematic reviews with meta-analyses evaluating positive and negative outcomes of hydroxychloroquine and chloroquine therapy," International Journal of Infectious Diseases, vol. 103, pp. 599-606, 2021. 